\title{
Aufbereitung von Wurzelkanälen
}

Für die Aufbereitung von Wurzelkanälen mit rotierenden Nickel-Titan-Instrumenten werden Elektromotoren mit speziellen Drehmomentsteuerungen empfohlen, um das Frakturrisiko niedrig zu halten. Dabei sind verschiedene Programme möglich, die das maximale Drehmoment begrenzen und dann den Motor abschalten oder in Linksdrehung wechseln. Eine weitere wichtige Komponente der endodontischen Therapie ist die Bestimmung und Einhaltung der Arbeitslänge, die vorzugsweise elektrometrisch und röntgenologisch geschieht. Der neue Endodontiemotor Nouvag Endo $\mathrm{V}$ vereinigt diese beiden Komponenten und soll im folgenden Erfahrungsbericht vorgestellt werden.

Der Endodontiemotor weist im Bedienpanel neben den Folientasten für die Einstellungen des Motors eine gut ablesbare LED-Anzeige für das Apexlocator- teil auf (Abb. 1). Mit den Folientasten werden die Funktionen des Motors eingestellt. Man kann hierbei zwischen Auto-Reverse, Auto-Stop und Slow Down wählen. Unseres Erachtens ist dabei die Einstellung „Auto-Reverse“ am ehesten sinnvoll. Das maximale Drehmoment ist mehr als ausreichend, das niedrigste als akzeptabel für fragile Instrumente $\mathrm{zu}$ bezeichnen. Die Besonderheit dieses Motors ist der eingebaute Apexlocator. Das sehr leichte und kompakte Handstück weist einen elektrischen Kontakt auf, der auf das jeweilige Wurzelkanalinstrument aufgeschoben wird.

Im klinischen Gebrauch arbeiteten die verschiedenen Komponenten des Gerätes zuverlässig. Positiv hervorzuheben ist das grazile und leichte Winkelstück und dessen ruhiger Lauf. Die Kontaktklemme am Winkelstückkopf erwies sich als ungünstig. Am Patienten lässt
Abb. 1 Das Bedienpanel des Endo V mit der Anzeige für den Apexlocator (rechts im Bild).

sich der Endo V ohne Probleme in die normale Routine eingliedern. Die Funktion des Motors und des Apexlocators ist insgesamt unproblematisch. Die akustische Anzeige der Eindringtiefe hat den Vorteil, dass der Motor nicht unbedingt im Sichtbereich aufgestellt werden muss. Bezüglich der klinischen Messgenauigkeit ist der Endodontiemotor absolut vergleichbar mit dem Root ZX.

\section{Korrespondenz}

O. A. Peters, E.Y. Chan

UCSF School of Dentistry,

Endodontic Division, 707 Parnassus Ave, San Francisco CA 94143-0758, USA

Dieser Beitrag ist entstanden mit freundlicher Unterstützung von Nouvag $\mathrm{GmbH}$, Konstanz. 